\title{
OPTIMIZATION ON SUPPLY-CONSTRAINED MODULE ASSEMBLY PROCESS
}

\author{
Jing $\mathrm{Liu}^{1}$, and Ming $\mathrm{Lu}^{2}$
}

\begin{abstract}
As off-shore prefabrication continues to gain momentum, supply chain management becomes increasingly complex for industrial modular construction projects and delays commonly occur to prefabricated modules. In order to make efficient utilization of limited module assembly resources (e.g., crews and bays) and reduce the waiting waste of materials on the module yard, a systematic optimization approach is desired to derive an optimal module assembly plan in coping with the dynamic supply chain and limited resource availability. By synthesizing information from the logistics management system, contract documents, and resources availability, a constraint programming based optimization algorithm is proposed. A case project abstracted from a real project is presented to demonstrate the feasibility and effectiveness of the proposed optimization approach. The information on module assembly start time, duration, and expected delivery time is generated to guide operations on the module yard. The minimum of total waiting time of materials on the module yard is derived for decision-making support. In conclusion, the proposed methodology seamlessly integrates principles of lean construction and constraints of resource scheduling into a constraint programming optimization formulation. This research potentially lends effective decision support to both crew work planning and materials logistical planning, ultimately leading to improvement on both construction productivity and logistical efficiency.
\end{abstract}

Keywords: Supply chain management, project scheduling, waiting waste reduction.

\section{INTRODUCTION}

To improve the construction industry performance, Ballard and Howell (1998) advocated the concept of lean construction, which is intended to turn the operation of construction industry into lean production while factoring in the unique characteristics of each construction process. Prefabrication and modularization is one typical case of lean construction. Over the past decade, mega processing projects have increasingly turned to pre-assembled modular construction in lieu of the conventional "stick build" construction. In Northern Alberta, Canada, the modular approach is generally adopted for executing mega oil sands projects, due to the benefits of reduction in construction cost and duration, along with improvement in quality and safety ( $\mathrm{Wu}$ and $\mathrm{Lu} 2013$ ).

The modular method separates the entire facility into multiple modular units in order to improve labor productivity and minimize on-site construction duration (Choi and Song 2014). For the modular construction method, each module, which is a basic building block, goes through off-site fabrication, transportation, and on-site installation. The balance among the three processes needs to be delicately maintained in order to

$1 \quad$ Ph.D. Student, Hole School of Construction Engineering, Department of Civil \& Environmental Engineering, University of Alberta, Edmonton, Alberta, Canada, jing.liu@ualberta.ca

2 Professor, Hole School of Construction Engineering, Department of Civil \& Environmental Engineering, University of Alberta, Edmonton, Alberta, Canada, mlu6@ualberta.ca 
minimize the waste of material, time and effort (Koskela et al, 2002). The concept of supply chain management (SCM), which was originated in manufacturing, aims to better satisfy the needs of end-customers and benefit all members in the chain (Walsh et al. 2004). However, due to productivity variability and unpredictable site demand, effective supply chain management is difficult to achieve in the construction industry compared to the manufacturing industry (Dubois and Gadde 2002; Tommelein et al. 2003). For the construction industry, instead of adopting a just-in-time (JIT) delivery method, the contractor tends to deliver the materials or pre-fabricated modules at the earliest time in order to mitigate the risk of late delivery (Tommelein et al. 2003). It would give rise to undesired wastes such as inventory waste, overproduction waste and waiting waste (Ohno, 1988).

Due to low labor cost and convenient sourcing of raw material (e.g. steel), the engineering design and fabrication of modules are usually undertaken by overseas vendors in East Asia, such as South Korea, China (Choi and Song 2014). The sizes of offshore manufactured modules generally fit into sea containers and satisfy road traffic regulations for shipping from the manufacturers in East Asia to oil sands projects in Alberta. Part of these off-shore manufactured sub-modules will be transported to the local module yard for further assembling into larger modules based on design specifications. Other small modules are transported to the site directly for installation. A module yard is a designated open space used to assemble and store modules. Module yards are typically divided into different work areas which are called as assembly bays. Each bay is usually occupied by only one module. The entire process of overseas industrial module fabrication is shown in Figure 1.

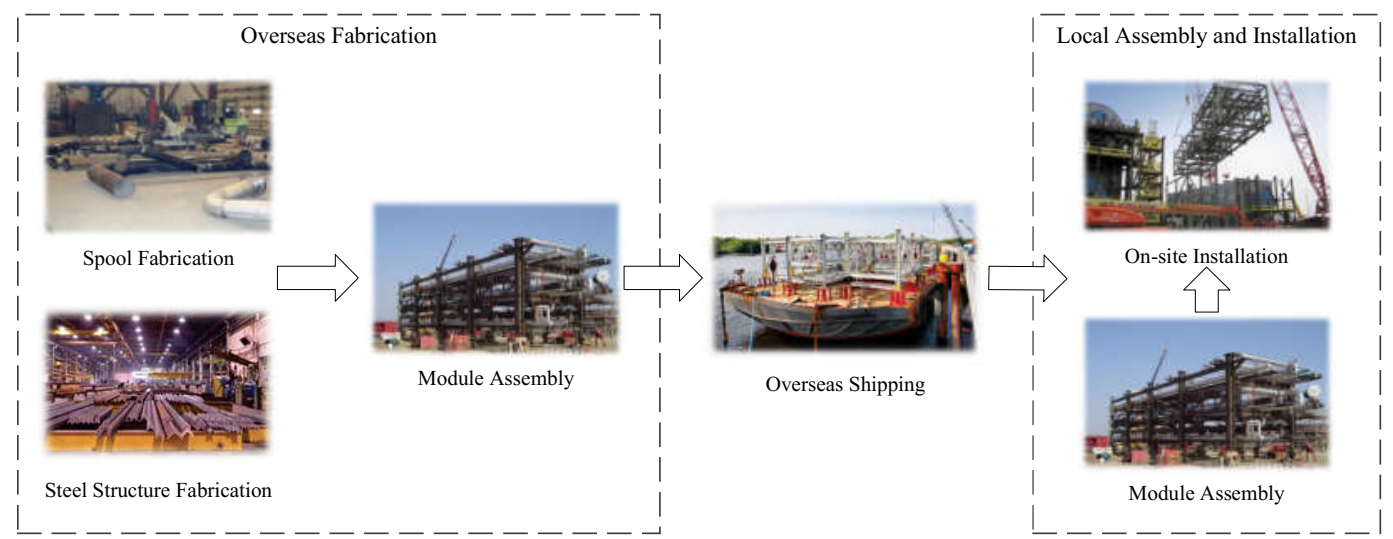

Figure 1: Overseas industrial module fabrication process

The overseas shipping process includes multiple stages of marine shipping, inland waterway shipping, and overland shipping. There are lots of uncertainties over the shipping process such as variable marine environment, the availability of transportation vehicle and local road regulations. The aforementioned supply chain management problems present risks to modular construction. Even if the module supply is planned based on on-site demands, unexpected interruptions to module fabrication and shipment can easily disrupt the original plan. In this circumstance, decisions should be made in order to mitigate the wastes potentially caused by the disrupted module supply, and in the meanwhile, the module assembly plan must meet on-site demands as closely as practically feasible. The unreliable supply of modules and the temporary nature of the module yard configuration make it difficult for project managers to update plans in a fast 
and effective manner. Furthermore, decisions resulting from subjective experience often turn out to be less efficient and non-optimal. For example, Liu and Lu (2016) demonstrated "improvised" schedule strategies based on common sense to mitigate material delay would actually increase the crews' idling time and lead to further project delay. Thus, a computer-aided optimization system is needed to derive and adjust the assembly plan in the module yard, aiming to mitigate the modules' waiting time on a module assembly yard. The revised module assembly plan is made according to the most updated shipping information of manufactured sub-modules.

In this paper, a constraint programming based optimization approach is presented to optimally plan assembly sequences in the module yard by using the supply information extracted from the logistics management system, the pre-set field erection deadlines extracted from construction contract and information of limited resources available in the module assembly yard. The aim is to minimize the total module-waiting-time on the module yard while ensuring on-time delivery of required modules to the downstream value-adding conversion activities in the field. This paper is organized as follows. In the following section, the constraint programming based optimization framework is presented to show the established model in terms of how to structure, formulate and solve the identified problem. Next, an illustrative case study is conducted to demonstrate the effectiveness of the proposed framework in scheduling the sequences of consolidating large modules by considering space and crew limits at the module assembly yard, and the arrival time and waiting time of each small module on the module yard. At the end, the conclusion is given to summarize the research.

\section{Constraint Programming Based Optimization Framework}

In the module yard, due to the limited availability of module assembly bays and the other installation constraints, some of these small modules, which are not to be assembled within 24 hours after their arrival, are stored in a laydown area. Thus, it will cause additional storing cost and handling cost without adding any value. Therefore, the total waiting time of all small modules is set as the objective function in order to evaluate the effectiveness of a module assembling plan. The smaller the waiting time is, the more effective is the module assembling plan. The waiting time of a small module is defined as the difference between the finish time of assembling the large module (when the small module becomes one part) and the arrival time of the small module.

The proposed constraint programming based optimization framework for module assembly is presented in Figure 2. There are four elements in this framework: data sources, extracted information, optimization engine and outputs for decision-support. To optimize the module assembly plan, three data sources are investigated for identifying constraints. They are 1) the logistics management database for overseas module supply information; 2) construction contract documents for on-site demand information, and 3) module yard crew information for the availability of labor resources. The mathematical modeling process for the three constraints will be explained in detail later.

After identifying the three constraints, they are entered into the optimization engine in search of the optimum solution resulting in the smallest waiting time averaged over all small modules. There are two outputs of the optimization engine. One output is the optimal assembly plan with the start time and finish time of consolidated large modules. The other one is the total waiting time for each small module, which provides a basis for calculating the additional logistics management cost (e.g., storage cost). 


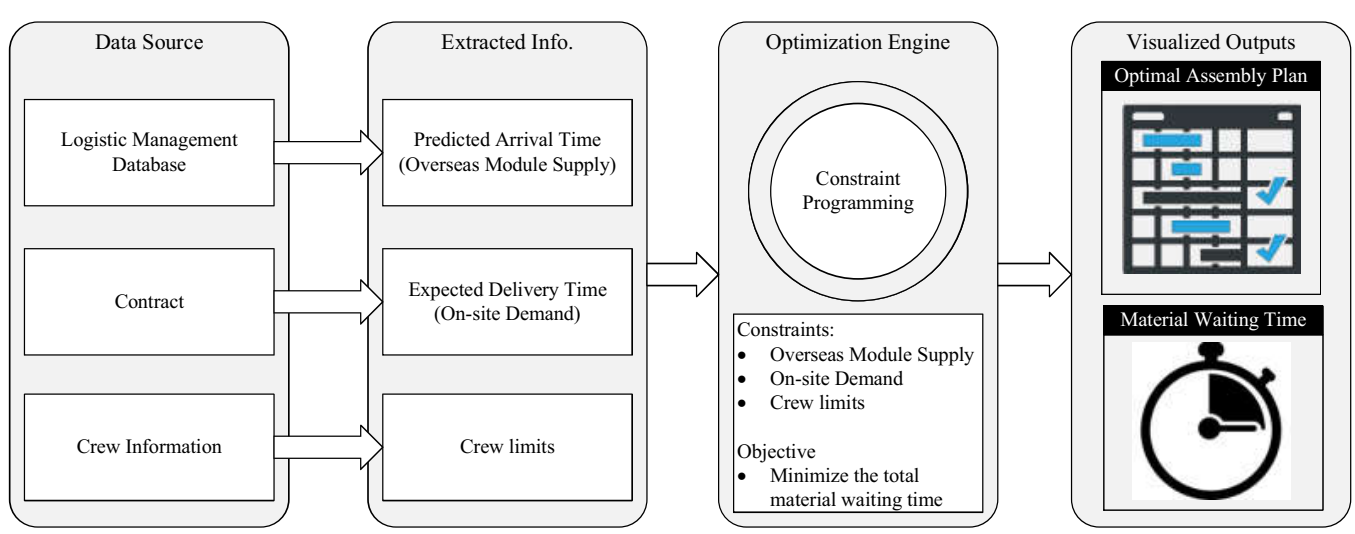

Figure 2: Industrial module construction process

\subsection{Formulation of the Mathematical Model}

Considering a module yard, there are $n$ large modules to be assembled using $m$ off-shore fabricated small modules. There are $R$ resources (i.e., crews and bays) available in the module yard. Each large module $j$ is composed by $k$ small modules. As described above, the objective function, which is to minimize the total waiting time of all small modules, can be expressed as Eq. (1).

$$
\min \left(\sum_{j=1}^{n} \sum_{i=1}^{k}\left(S F_{j}-T_{i, j, \text { arrive }}\right)\right)
$$

where, $S F_{j}$ is the scheduled finish time of the large module $j ; T_{i, j, \text { arrive }}$ is the arrival time of the small module $i$, which is one part of the large module $j$.

Furthermore, the arrival time $T_{i, j, \text { arrive }}$ of small module $i$ puts one hard constraint on the start time $S S_{j}$ for assembling the large module $j$, namely, the large module $j$ cannot be started before all required $k$ small modules have arrived at the module yard. The starttime constraint is expressed as Eq. (2).

$$
S S_{j} \geq \max \left(T_{i, j, \text { arrive }}\right) ; \quad i=1, \cdots, k ; j=1, \cdots, n
$$

On the other hand, the expected delivery time $T_{j, \text { deliver }}$ of large module $j$ limits its finish time $S F_{j}$, which equals to the summation of the scheduled start time $S S_{j}$ and the duration $D_{j}$. It means the large module $j$ has to be finished before the expected delivery time. The constraint is shown in Eq. (3)

$$
S F_{j}=S S_{j}+D_{j} \leq T_{j, \text { deliver }}, j=1, \cdots, n
$$

The crew limit $R$ puts another hard constraint on the number of crews that can be employed in any period. Thus, the resource constraint can be expressed as Eq. (4)

$$
\sum_{j \in M_{0} d_{t}} R_{j} \leq R, t=1, \cdots, D
$$

where $R_{j}$ is the required crew amount for assembling large module $j ; \operatorname{Mod}_{t}$ is the set of large modules which are already started but not finished or ready to be started at time $t ; D$ is the total duration for assembling the $n$ large modules. Note all the time related 
variables except for duration variables (i.e., activity duration $D_{j}$ and total assembling duration $D$ ) refer to time points with the start time being zero.

The mathematical model, which is shown as the Eq.(1), Eq.(2), Eq.(3) and Eq.(4), can be readily solved by constraint programming. Constraint programming is a hybrid method integrating multiple techniques in operations research (OR), artificial intelligence (AI) and graph theory (Rossi et al. 2006). It is much effective for solving complicated scheduling problems (Bockmayr and Hooker 2003). For example, Liu and Wang (2012) employed constraint programming to enhance the computation efficiency for scheduling linear construction projects with multi-skilled crews. Menesi et al. (2013) demonstrated the capability of constraint programming to handle the time-cost trade-off problem for large-scale projects involving thousands of activities. It is worth mentioning that IBM ILOG CPLEX Optimization Studio V12.6 (2016) provides a powerful toolkit for performing constraint programming and optimization, which has been utilized as the computational platform in construction scheduling research (Menesi et al. 2013; Siu et al. 2015). Hence, in this study, it was used as the computing tool to solve the constraint programming formulation and search for the optimum solution to the defined problem.

\section{CASE STUDY}

In this section, a case project is used to illustrate the effectiveness of the proposed methodology. The data and information for this case were prepared based on a modular construction project in Alberta, Canada. Assume 10 large modules in total are to be assembled. Three assembly bays and three crews are allocated to assemble the 10 large modules. For each large module, it is composed by 4 small sub-modules. 40 small modules are manufactured off-shore. And then each small module is packed in a sea container for overseas shipping. Due to unreliable supply process and limited assembly bay resources, the large module is only scheduled to start with assembly after all required 4 containers arrive at the module yard. Table 1 lists the required container IDs for each large module.

Table 1: Required container ID for each large module

\begin{tabular}{|c|c|c|c|c|c|c|c|c|c|c|c|c|c|c|c|c|}
\hline Module ID & \multicolumn{4}{|l|}{ M1 } & \multicolumn{4}{|c|}{ M2 } & \multicolumn{4}{|l|}{ M3 } & \multicolumn{4}{|c|}{ M4 } \\
\hline Required Container ID & 1 & 6 & 8 & 15 & 5 & 16 & 22 & 26 & & 24 & 30 & 32 & 3 & 9 & 11 & 17 \\
\hline Module ID & M5 & & & & M6 & & & & M7 & & & & M8 & & & \\
\hline Required Container ID & 7 & 10 & 13 & 14 & 2 & 4 & 12 & 18 & 21 & 27 & 34 & 39 & 20 & 28 & 35 & 40 \\
\hline Module ID & M9 & & & & M1 & & & & & & & & & & & \\
\hline Required Container ID & 23 & 33 & 36 & 38 & 25 & 29 & 31 & 37 & & & & & & & & \\
\hline
\end{tabular}

Typically, the layout of assembly bays in a module yard changes over time as the type of modules being fabricated changes, and thus some preparation work is needed before starting to assemble one module such as changing the layout and installing structural blocks to support modules. Thanks to the logistics management system, the expected arrival time of each small module is available, the preparing work can be 
finished before small modules arrive at the module yard. Thus, in the case study, it is ignored. The assembly of one large module can be scheduled on the same day with the arrival time of the last required container. In this section, different scenarios with different arrival times of small modules are assumed to simulate the effectiveness of the proposed optimization approach in coping with the uncertainties in the module supply chain, while minimizing the wastes in terms of "idling" materials as per the lean principle. The arrival times for each scenario is shown in Table 2. Scenario 2 can be treated as an update of Scenario 1: some containers are delayed due to unexpected disruptions.

Table 2: Arrival time of each small module in different scenarios

\begin{tabular}{cccccccccccccccc}
\hline Container ID & $\mathbf{1}$ & $\mathbf{2}$ & $\mathbf{3}$ & $\mathbf{4}$ & $\mathbf{5}$ & $\mathbf{6}$ & $\mathbf{7}$ & $\mathbf{8}$ & $\mathbf{9}$ & $\mathbf{1 0}$ & $\mathbf{1 1}$ & $\mathbf{1 2}$ & $\mathbf{1 3}$ & $\mathbf{1 4}$ & $\mathbf{1 5}$ \\
\hline Scenario 1 & 0 & 2 & 3 & 4 & 5 & 5 & 6 & 7 & 7 & 7 & 7 & 8 & 9 & 9 & 9 \\
Scenario 2 & 0 & 2 & 3 & 4 & 5 & 5 & 6 & 9 & 9 & 9 & 9 & 8 & 9 & 9 & 9 \\
\hline Container ID & $\mathbf{1 6}$ & $\mathbf{1 7}$ & $\mathbf{1 8}$ & $\mathbf{1 9}$ & $\mathbf{2 0}$ & $\mathbf{2 1}$ & $\mathbf{2 2}$ & $\mathbf{2 3}$ & $\mathbf{2 4}$ & $\mathbf{2 5}$ & $\mathbf{2 6}$ & $\mathbf{2 7}$ & $\mathbf{2 8}$ & $\mathbf{2 9}$ & $\mathbf{3 0}$ \\
\hline Scenario 1 & 9 & 10 & 11 & 11 & 12 & 12 & 13 & 14 & 14 & 14 & 15 & 15 & 16 & 16 & 16 \\
Scenario 2 & 9 & 13 & 11 & 11 & 12 & 12 & 13 & 14 & 14 & 14 & 15 & 15 & 20 & 20 & 20 \\
\hline Container ID & $\mathbf{3 1}$ & $\mathbf{3 2}$ & $\mathbf{3 3}$ & $\mathbf{3 4}$ & $\mathbf{3 5}$ & $\mathbf{3 6}$ & $\mathbf{3 7}$ & $\mathbf{3 8}$ & $\mathbf{3 9}$ & $\mathbf{4 0}$ & & & & & \\
\hline Scenario 1 & 17 & 17 & 18 & 18 & 19 & 19 & 19 & 20 & 20 & 21 & & & & \\
Scenario 2 & 17 & 17 & 20 & 20 & 19 & 19 & 19 & 20 & 20 & 21 & & & & & \\
\hline
\end{tabular}

The expected delivery time for each large module is extracted from the contract document and listed in Table 3. The duration needed for assembling each large module is shown in Table 3 as well. Preparation tasks need to be done prior to loading the finished module onto a truck, such as fixing lugs for lifting and lashing for fixing the module on the truck. Thus, the module is required to be finished one day before the expected delivery time. For example, module M1 is expected to be delivered on Day 15, so it must be finished by Day 14 .

With all the available information, the proposed optimization approach was readily applied to schedule the assembly sequence of the 10 large modules in search of the shortest total waiting time of all small modules subject to available resources, which include two crews and two assembly bays. In the meantime, the start time for assembling one large module should be no earlier than the last arrival time of the required containers, and the finish time should be one day before the expected delivery time. The optimized start time and finish time of each module for different scenarios are listed in Table 3. The total waiting time for all small modules is 298 days and 311 days for Scenario 1 and Scenario 2. The total waiting time increases due to the delay of some containers, which delays the start time for assembling the corresponding large modules, thus increasing the idling time of arrived small modules in the module yard. The proposed method can provide the plan with the least total waiting time. The related storage cost can also be derived for supporting the decision-making process.

For an actual project, the developed optimization algorithm can be run multiple times over the project duration in order to mitigate the potential increase of module waiting 
wastes given the most recent module supply information. Under the fast-changing logistical conditions, the proposed methodology can provide effective assistance for project managers to make and update module assembly plans, aimed to reduce the waiting waste of materials.

Table 3: Detailed schedule information for each large module

\begin{tabular}{|c|c|c|c|c|c|c|}
\hline \multirow{2}{*}{$\begin{array}{l}\text { Module } \\
\text { ID }\end{array}$} & \multirow{2}{*}{$\begin{array}{c}\text { Assembly } \\
\text { Duration } \\
\text { (day) }\end{array}$} & \multirow{2}{*}{$\begin{array}{c}\text { Expected } \\
\text { Delivery } \\
\text { Time }\end{array}$} & \multicolumn{2}{|c|}{ Scenario 1} & \multicolumn{2}{|c|}{ Scenario 2} \\
\hline & & & $\begin{array}{l}\text { Scheduled } \\
\text { Start Time }\end{array}$ & $\begin{array}{l}\text { Scheduled } \\
\text { Finish Time }\end{array}$ & $\begin{array}{l}\text { Scheduled } \\
\text { Start Time }\end{array}$ & $\begin{array}{l}\text { Scheduled } \\
\text { Start Time }\end{array}$ \\
\hline M1 & 5 & 15 & 9 & 14 & 9 & 14 \\
\hline M2 & 3 & 20 & 15 & 18 & 15 & 18 \\
\hline M3 & 4 & 25 & 17 & 21 & 20 & 24 \\
\hline M4 & 3 & 18 & 10 & 13 & 13 & 16 \\
\hline M5 & 2 & 13 & 9 & 11 & 9 & 11 \\
\hline M6 & 3 & 16 & 11 & 14 & 11 & 14 \\
\hline M7 & 5 & 31 & 20 & 25 & 24 & 29 \\
\hline M8 & 4 & 29 & 24 & 28 & 24 & 28 \\
\hline M9 & 4 & 26 & 21 & 25 & 20 & 24 \\
\hline M10 & 5 & 28 & 19 & 24 & 20 & 25 \\
\hline
\end{tabular}

\section{CONCLUSIONS}

To improve construction efficiency, prefabrication and modularization are implemented for most industrial construction projects. However, with more and more modules prefabricated overseas, the supply chain of the industrial modular construction becomes increasingly complicated and uncertain. Module supply delay is commonly encountered in the module pre-fabrication and shipping processes, which would disrupt the ensuing module assembly plans. Module yard assembly, which is actually part of the material flowing process, precedes the final installation on site, represents the value-adding conversion activity according to the lean concept. Therefore, the total module-waitingtime on the module yard needs to be well planned and controlled such that all the "Mura" (i.e., uncertainty/inconsistency) along the flowing process prior to the module assembly yard are accommodated. In the meanwhile, the "Muda" (i.e., waiting time) at the yard is best controlled in order to feed materials to the execution of value-adding conversion activities in field erection.

In this paper, an optimization framework for implementing constraint programming is proposed to mitigate the increase on total waiting time of materials caused by uncertain module supply processes. The framework consists of (1) data sources, including contract documents, logistics management system and crew hiring documents; (2) extracted information including contractual deadline, time-dependent module supply patterns and resources availability; (3) constraint programming based optimization 
engine; and (4) analytical outputs in terms of the detailed schedule of each large module and the total waiting time of small modules. Information and data were extracted from a real modular construction project in order to build a case study for demonstration and validation of the proposed framework. The resulting schedule for assembling 10 large modules is presented. The least total waiting time for small modules is derived providing a basis for calculating the corresponding storage cost. In conclusion, the proposed methodology can assist practitioners in (1) interpretation of material supply data and project scheduling data in an integrative fashion and (2) generation of the best crew job plan resulting in the least material waiting time incurred at the module assembly yard.

\section{REFERENCES}

Ballard, Glenn, and Greg Howell. "What kind of production is construction." Proc. 6th Annual Conf. Int'l. Group for Lean Construction. 1998.

Bockmayr, A., and Hooker, J. N. (2003). "Constraint programming." Handbook of Discrete Optimization (Eds. K. Aardal, G. Nemhauser, and R. Weismantel).

Choi, J., \& Song, H. (2014). Evaluation of the modular method for industrial plant construction projects. International Journal of Construction Management, 14(3), 171180.

Dubois, A., and Gadde, L.-E. (2002). "The construction industry as a loosely coupled system: implications for productivity and innovation." Construction Management \& Economics, 20(7), 621-631.

IBM ILOG CPLEX Optimization Studio V12.6. 2016. [Computer software]. IBM, Armonk, NY.

Koskela, Lauri, et al. "The foundations of lean construction." Design and construction: Building in value (2002): 211-226.

Liu, J., and Lu, M. (2016). "Proposing a material-driven recovery schedule optimization framework: spool fabrication case "16th International Conference on Computing in Civil and Building Engineering (ICCCBE) Osaka, Japan.

Liu, S.-S., and Wang, C.-J. (2012). "Optimizing linear project scheduling with multiskilled crews." Automation in Construction, 24, 16-23.

Menesi, W., Golzarpoor, B., and Hegazy, T. (2013). "Fast and near-optimum schedule optimization for large-scale projects." Journal of Construction Engineering and Management, 139(9), 1117-1124.

Ohno, Taiichi (1988), Toyota Production System: Beyond Large-Scale Production, Productivity Press, ISBN 0-915299-14-3

Rossi, F., van Beek, P., and Walsh, T. (2006). Handbook of Constraint Programming, Elsevier Science.

Siu, M.-F. F., Lu, M., and AbouRizk, S. (2015). "Zero-One Programming Approach to Determine Optimum Resource Supply under Time-Dependent Resource Constraints." Journal of Computing in Civil Engineering, 04015028.

Tommelein, I.D., Walsh, K.D., and Hershauer, J.C. (2003), "Capital projects supply chain management." Research Rep. No. 172-11, Construction Industry Institute, Austin.

Walsh, Kenneth D., et al. "Strategic positioning of inventory to match demand in a capital projects supply chain." Journal of Construction Engineering and Management 130.6 (2004): 818-826.

Wu, T. and Lu, M. 2013. Modularization Program Execution Optimization on Mega Oil Sands Projects. AACE INTERNATIONAL TRANSACTIONS CSC.1285.1:28. 